\title{
1,4-Butanediol Content of Aqua Dots Children's Craft Toy Beads
}

\author{
Jeffrey R. Suchard, $M D^{a}$, Sergey A. Nizkorodov, PhD ${ }^{b}$, Stacy Wilkinson ${ }^{b}$
}

aDepartment of Emergency Medicine, University of California, Irvine Medical Center, Orange, CA

bDepartment of Chemistry, University of California, Irvine, Irvine, CA

\begin{abstract}
Introduction: The U.S. Consumer Product Safety Commission announced a recall of Aqua Dots (Spin Master Ltd.; Toronto, Canada) on November 7, 2007 due to children becoming ill after swallowing beads from these toy craft kits. Reports suggested that the beads contained 1,4-butanediol (1,4-BD), a precursor to gamma-hydroxybutyrate (GHB), rather than the intended, but more expensive 1,5-pentanediol (1,5-PD). We measured the 1,4-BD and 1,5-PD content of Aqua Dots beads to determine if 1,5-PD had been completely substituted with 1,4-BD by the manufacturer, and if the reported clinical effects from swallowing Aqua Dots beads were consistent with the estimated ingested 1,4-BD dose.

Methods: In vitro bench research using gas chromatography-mass spectroscopy (GC-MS) was performed. Dilute samples of pure 1,4-BD and 1,5-PD in water were used for the calibration of the GC-MS instrument. We then soaked Aqua Dots beads in water for varying durations, and the resultant solutions were analyzed for 1,4-BD and 1,5-PD content.

Results: Aqua Dots beads weighed $79.3 \mathrm{mg}$ each $( \pm 0.6 \mathrm{mg}, \mathrm{SD})$, and contained $13.7 \%$ ( $\pm 2.4 \%$, SD) 1,4 -BD by weight; this corresponds to a $1,4-\mathrm{BD}$ content of $10.8 \mathrm{mg}( \pm 1.9 \mathrm{mg}, \mathrm{SD})$ per bead. No 1,5-PD was detected in any beads.

Conclusions: Aqua Dots beads contained a surprisingly high amount (nearly 14\%) of extractable 1,4-BD. No 1,5-PD was detected, corroborating reports that this chemical had been completely replaced with a substitute that is metabolized into GHB after ingestion. Reports of ataxia, vomiting, seizure activity, and self-limited coma in children are consistent with the ingestion of several dozen Aqua Dots beads.
\end{abstract}

\section{INTRODUCTION}

Aqua Dots (Spin Master Ltd.; Toronto, Canada) was a popular children's craft toy kit. The kits included small colored plastic beads that would fuse together when sprayed with water and then allowed to dry, forming various multidimensional designs. In 2007, Aqua Dots was listed among Toy Wishes magazine's "Hot Dozen" top toys and was named the "Toy of the Year" in Australia, where it was marketed under the name Bindeez [1,2]. More than 12 million craft kits, containing over 8 billion beads, were sold worldwide [3]. An estimated 4.2 million units were distributed in the United States alone between April and November 2007 [4].

Keywords: 1,4-butanediol, gamma-hydroxybutyrate, poisoning, toy

Notes: The information in this study was presented previously in the following venues: Western Regional Society for Academic Emergency Medicine Research Forum, Santa Ana, CA, March 28, 2008; and Society for Academic Emergency Medicine Annual Meeting, Washington, DC, May 29, 2008.

There was no outside funding of any kind used for this study.

Corresponding Author: Jeffrey R. Suchard, MD, UCIMC, Dept of Emergency Medicine, 101 The City Drive, Route 128, Orange, CA 92868. E-mail: jsuchard@uci.edu 
In late 2007, several reports of children developing vomiting, ataxia, and self-limited coma after swallowing Aqua Dots beads appeared in the Australian and American media [2,5-7]. Gammahydroxybutyrate (GHB) was detected in biologic samples from some of these children [8,9]. The U.S. Consumer Product Safety Commission (CPSC) announced a voluntary recall of Aqua Dots on November 7, 2007, and the product similarly was recalled or banned in other countries [2-4]. Further investigation found that the Chinese bead manufacturer had replaced 1,5-pentanediol (1,5-PD), a common ingredient in various glues, with 1,4-butanediol (1,4-BD) $[3,5]$. The substitution may have occurred because 1,4-butanediol cost considerably less than 1,5-pentanediol: US $\$ 1350-\$ 2800$ per metric ton vs. \$9700 [7]. 1,4-Butanediol is metabolized in vivo into GHB [10].

Our objective was to measure the 1,5-PD and 1,4-BD content of Aqua Dots beads to determine if 1,4-BD had been completely substituted for 1,5-PD by the manufacturer and if the reported clinical effects from swallowing Aqua Dots beads were consistent with the estimated 1,4-BD dose in cases in which the number of beads ingested was reported.

\section{MATERIALS AND METHODS}

One of the authors (JRS) purchased an Aqua Dots Starter Pen Pack through an online distributor on November 12, 2007. Before filling the order, the distributor contacted the purchasing author to ensure that he knew the product had been recalled, but released the shipment when the proposed investigation was explained and because CPSC recalls are voluntary and thus do not make the sale of recalled products illegal.

The toy craft kit contained 500 beads $\sim 5.0 \mathrm{~mm}$ in diameter in 6 colors: black, blue, orange, green, yellow, and red. Multiple beads were weighed to establish an average weight of $79.3 \mathrm{mg} \pm 0.6 \mathrm{mg}$ (standard deviation). There was no statistically significant variation in weight among beads of different colors.

\section{Determination of the Presence of 1,4-Butanediol and/or 1,5-Pentanediol in Aqua Dots Beads}

For each color, 2 beads were placed into 50-mL deionized water (18 $\mathrm{M} \Omega$ ) to partially dissolve them. After 2 hours dissolution, a sample of each solution was transferred using a syringe filter into 1-mL gas chromatography/mass spectroscopy (GC/MS) vials sealed with Teflon-coated septa. Calibration solutions were prepared containing known concentrations of 1,4-BD (Sigma Aldrich, St. Louis, MO; Reagent Plus grade, >99\%) and 1,5-PD (Acros Organics, Geel, Belgium; 98\%) in deionized water.

Chromatograms were acquired using the ThermoFinnigan Trace GC 2000 instrument equipped with Restek Rtx-65 column (Thermo Fisher Scientific, Inc., Waltham, MA). The instrument was interfaced to ThermoFinnigan Trace MS 2000 mass spectrometer and ThermoFinnigan AS 2000 autosampler. The relevant experimental parameters were: $230^{\circ} \mathrm{C}$ injector port temperature; $1.0-1.5 \mu \mathrm{L}$ injection volume; $230^{\circ} \mathrm{C}$ MS source temperature. The temperature program for the column started from $40^{\circ} \mathrm{C}$ followed by $8-16^{\circ} \mathrm{C} / \mathrm{min}$ ramp until $220^{\circ} \mathrm{C}$, followed by 2 -minute hold, and cooling. The mass spectrometer was programmed to turn on after 2.5 minutes (to avoid the $\mathrm{H} 2 \mathrm{O}$ solvent peak) and record total ion current (TIC) for ions in the 35-200 Dalton range.

Liquid phase nuclear magnetic resonance (NMR) was also used to test for the presence of 1,4-BD and 1,5-PD in the beads. NMR additionally allowed for determination of whether there were any other small molecules in solution that were missed by GC/MS. Two beads were soaked in $50-\mathrm{mL}$ deuterated water $\left(\mathrm{D}_{2} \mathrm{O}\right)$ for several hours. After that, the solution was filtered, placed in a standard 5-mm NMR tube, and analyzed with a Bruker DRX $500 \mathrm{MHz}$ NMR spectrometer (Bruker Optics, Inc., Billerica, MA). ${ }^{1} \mathrm{H}$ spectra with solvent suppression and ${ }^{13} \mathrm{C}$ spectra with ${ }^{1} \mathrm{H}$ decoupling were recorded and compared to the corresponding spectra of 1,4-BD and 1,5-PD reference solutions in $\mathrm{D}_{2} \mathrm{O}$. The chemical shifts were referenced to the DSS NMR standard.

\section{Time-dependent Release of 1,4-Butanediol from Aqua Dots Beads}

To determine the rate at which the beads released 1,4-BD and the maximal amount of 1,4-BD extractable in solution, time-resolved experiments were conducted. Twelve beads ( 2 beads of each color) were placed into a temperature-controlled beaker containing 1000-mL deionized water. Because the amount of extractable 1,4$\mathrm{BD}$ may have been temperature-dependent, this experiment was conducted one time at room temperature, and a second time at $40^{\circ} \mathrm{C}$ to represent the maximum expected human body temperature. The solutions were continuously stirred, and small aliquots $(<1 \mathrm{~mL})$ were withdrawn at time intervals and transferred into GC/MS vials for subsequent analysis. The samples were withdrawn through a syringe filter to avoid getting debris from the partially dissolved beads into the GC/MS instrument. This step was necessary as the beads initially became discolored and swollen, then slowly disintegrated, leaving the solution cloudy with tiny colorless particles.

Calibration solutions of $1,4-\mathrm{BD}$ in water were prepared with concentrations ranging from $0 \mathrm{~mol} / \mathrm{L}$ to $0.003 \mathrm{~mol} / \mathrm{L}$. The chromatograms for the calibration solutions and test samples were recorded on the same day under identical conditions. The peaks corresponding to 1,4 -BD were integrated to calculate the concentrations in all the samples as a function of time. The concentrations were then converted into the time-dependent fraction, $F(t)$, of the bead mass extracted into the solution as follows,

$$
F(t)=\frac{[1,4 \text {-butanediol }] \times \mathrm{V} \times \mathrm{MW}}{\text { mass of beads }} \times 100 \%
$$

where [1,4-butanediol] is the measured concentration in mol/L, $\mathrm{V}$ is the volume of solution $(1.0 \mathrm{~L}), \mathrm{MW}$ is the molecular weight 
of 1,4-BD (90.12 $\mathrm{g} / \mathrm{mol})$, and mass is the combined mass of the beads placed into the solution.

\section{RESULTS}

\section{Determination of the Presence of 1,4-Butanediol and/or 1,5-Pentanediol in Aqua Dots Beads}

Figure 1 shows sample chromatograms of an Aqua Dots extract compared to that of the reference solution. The peaks eluting at 6.7 minutes and 7.8 minutes from the reference solution correspond to 1,4-BD and 1,5-PD, respectively. The asymmetric tailing shape of the GC peaks is common in chromatographic separation of alcohols. The assignments were explicitly confirmed by comparing the mass spectra to the National Institute of Standards and Technology mass-spectrometric library.

It is clear that 1,4-BD, but not 1,5-PD, was extractable from the Aqua Dots beads under the experimental conditions. Almost no interfering substances were co-extracted from the beads. No trace of 1,5-PD was detected in this or any subsequent test. Approximately the same amounts of 1,4-BD were observed for all samples, with no significant variation between different bead colors.

Two major peaks appeared in the ${ }^{13} \mathrm{C}$ NMR spectrum of solution obtained by soaking the beads in $\mathrm{D}_{2} \mathrm{O}$ : $61.6 \mathrm{ppm}$ (from the terminal carbon atoms in 1,4-BD) and $27.9 \mathrm{ppm}$ (from the $\mathrm{C} 2$ and $\mathrm{C} 3$ carbons in 1,4-BD). The same peaks were observed in the spectrum of the 1,4-BD standard dissolved in $\mathrm{D}_{2} \mathrm{O}$. No detectable peaks corresponding to 1,5-PD were observed, confirming the results obtained by the GC-MS method. Furthermore, the ${ }^{13} \mathrm{C}$ spectrum contained no significant additional peaks, suggesting that $1,4-\mathrm{BD}$ was the major soluble organic component of the beads. The ${ }^{1} \mathrm{H}$ spectrum contained several weak, broad peaks at $1.7,4.1$, and $8.7 \mathrm{ppm}$, which did not belong to 1,4-BD and did not correlate to any of the observed carbon peaks. These peaks likely came from unidentified polymeric compounds in the beads.

\section{Time-dependent Release of 1,4-Butanediol from Aqua Dots Beads}

Figure 2 shows the time dependence of the fraction of 1,4-BD extracted from the beads into the solution. At both temperatures, the dissolved faction reached a steady state on a time scale of several hours. The time-dependent fraction was fitted with the following function:

$$
F(t)=F_{\max } \times\left(1-e^{-t / \tau}\right)
$$

where $F_{\max }$ is the maximal faction of 1,4 -BD that can be extracted from the beads and $\tau$ is the characteristic rise time for dissolution. The fits are shown in Figure 2 as solid lines, and the resulting fit parameters are given in Table 1 . Increasing the solution temperature from $20^{\circ} \mathrm{C}$ to $40^{\circ} \mathrm{C}$ accelerated the rate of dissolution by about $40 \%$. Half of the maximal 1,4-BD was extracted by 97 minutes at $20^{\circ} \mathrm{C}$, and by 69 minutes at $40^{\circ} \mathrm{C}$.

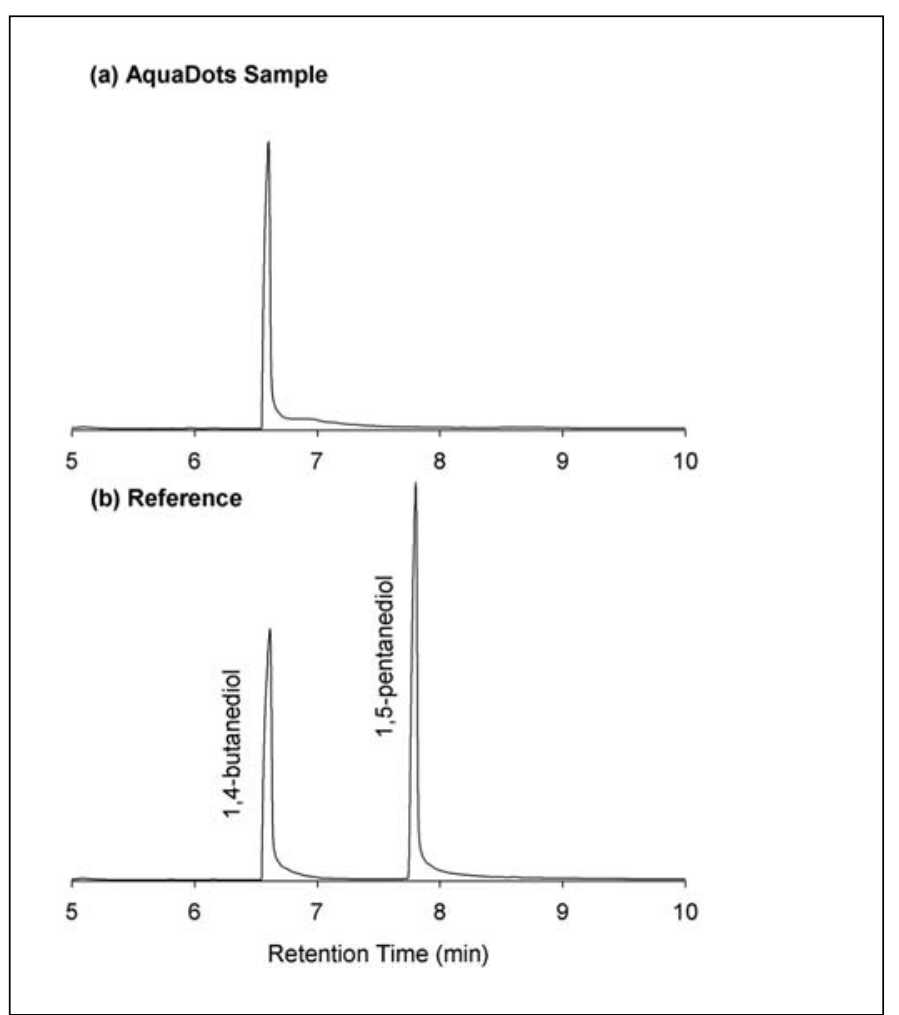

Figure 1: Sample GC/MS traces of an Aqua Dots sample and a reference solution containing 1,4-BD and 1,5-PD. Only 1,4-BD is observed in the beads.

The aggregate data showed that $13.7 \pm 2.4 \%$ of the bead weight could be extracted into solution as 1,4-BD. Given the bead weight of $79.3 \pm 0.6 \mathrm{mg}$, this corresponds to a 1,4-BD content of $10.8 \pm 1.9 \mathrm{mg}$ per bead.

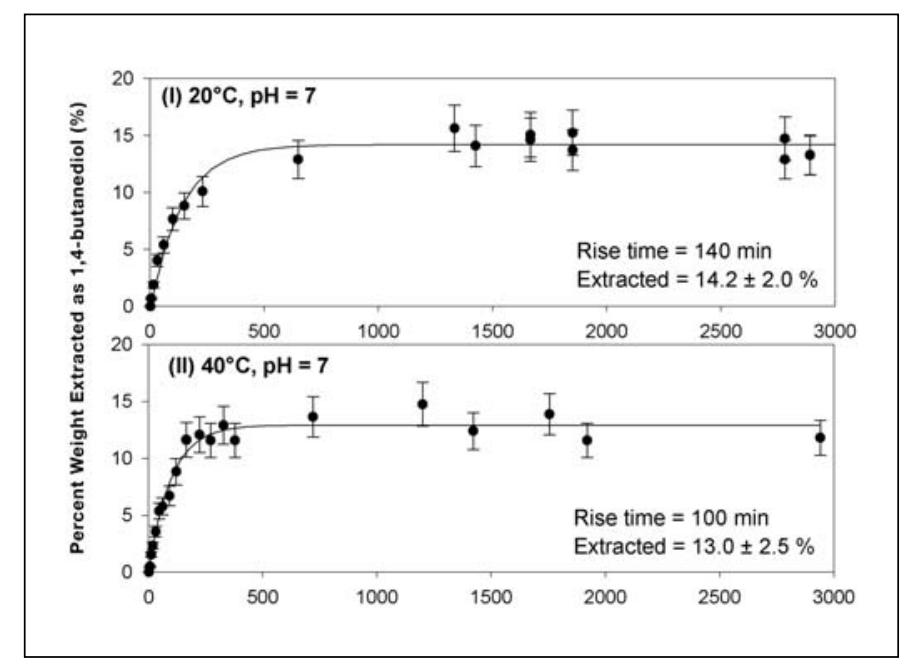

Figure 2: Time dependence of 1,4-BD extraction from Aqua Dots beads into solution, as a percent of total bead weight. 
Table 1: Summary of Experimental Conditions and Results from 2 Dissolution Experiments

\begin{tabular}{lcccc}
\hline Experiment & $\begin{array}{c}\text { Beads } \\
(\mathbf{g})\end{array}$ & $\mathbf{T}\left({ }^{\circ} \mathbf{C}\right)$ & $\begin{array}{c}\text { Rise time } \\
([\mathbf{t}], \mathbf{m i n})\end{array}$ & $\begin{array}{c}\text { Final fraction } \\
\text { of dissolved } \\
\text { 1,4-butanediol (\%) }\end{array}$ \\
\hline I & 0.942 & 20 & 140 & 14.2 \\
\hline II & 0.938 & 40 & 100 & 13 \\
\hline
\end{tabular}

\section{DISCUSSION}

When assessing the expected toxic effects of ingesting Aqua Dots beads, it becomes necessary to consider 1,4-BD in comparison to its closest chemical congeners, GHB and sodium 4-hydroxybutyrate (Figure 3). These chemicals share a common 4-carbon backbone and terminal (gamma) hydroxyl group, differing only at the \#1 carbon. On a molecule-for-molecule basis, these chemicals will exert the same clinical effects, barring the delay necessary for oxidative metabolism of 1,4-BD into GHB; but they are not equivalent on a gram-for-gram basis, since they have differing molecular weights. Each gram of 1,4-BD should be pharmacologically equivalent to $1.155 \mathrm{~g} \mathrm{GHB}$ and $1.399 \mathrm{~g}$ sodium 4-hydroxybutyrate due to these differences.

Much of the available dose-response data for GHB derive from spontaneous and often nonvalidated reports related to illicit use, and are therefore not entirely reliable. Dose-response data for 1,4BD are similarly lacking, and those reports related to its illicit use typically fail to distinguish it from GHB. Notwithstanding these limitations, some general comments can be made regarding the

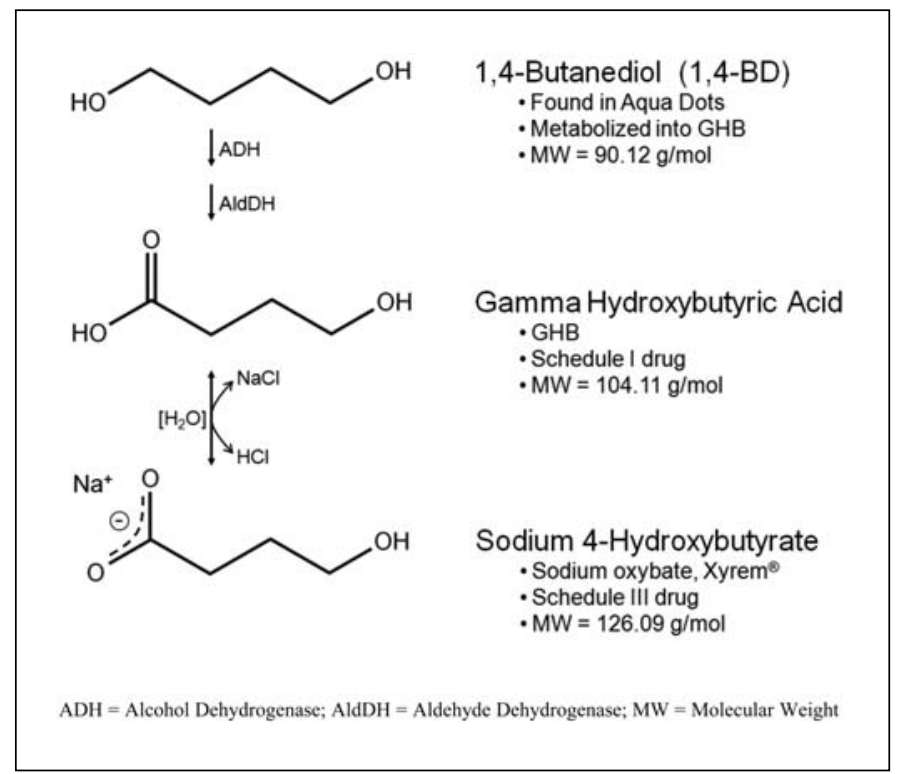

Figure 3: Relationship between 1,4-butanediol and its closest chemical congeners. $A D H, A l d D H$, and MW. dose-response curve of GHB and/or 1,4-BD. The Vaults of Erowid website states that "a standard recreational dose of pure GHB powder is between 1-3 g" [11]. Low doses (0.5-1.5 g) cause effects similar to 1-3 alcoholic drinks with mild relaxation and dizziness, medium doses (1.0-2.5 g) can produce positive mood changes and nausea, while heavy doses ( $>2.5 \mathrm{~g}$ ) produce euphoria, disequilibrium, and vomiting [12]. Doses between 3-5 g "can induce heavy sleep," while the typical dose range for an "overdose" is 5-10 g [13]. Overdoses of GHB are described as causing nausea, dizziness, vomiting, and a "temporarily unarousable sleep (sometimes characterized as a type of coma) for 1-4 hours" and may be caused by doses as low as $2 \mathrm{~g}$ depending on body weight and individual sensitivity [12]. The clinical effects from Aqua Dots bead ingestions reported in the lay press and in some medical journals would be classified as "overdoses" by Erowid's working definition $[2,5-9,14]$. The typical corresponding dose of 5-10 $\mathrm{g}$ in a $70-\mathrm{kg}$ adult converts to $71-142 \mathrm{mg} \mathrm{GHB} / \mathrm{kg}$, or to $62-124 \mathrm{mg} 1,4-\mathrm{BD} / \mathrm{kg}$.

Sodium 4-hydroxybutyrate has some more reliable doseresponse data, as it has been systematically studied and is FDA approved (Xyrem [sodium oxybate]; Jazz Pharmaceuticals, Palo Alto, CA) as a Schedule III drug under the Controlled Substances Act for treating cataplexy and excessive daytime sleepiness in narcolepsy [15]. Per the drug's package insert, therapeutic dosing of sodium oxybate is $4.5 \mathrm{~g} / \mathrm{night}$ in 2 divided doses. Patients are advised to take both doses while already in bed, to prepare the second dose before the first is taken, and to set an alarm to awaken for the second dose because the first may induce sleep. Two study subjects developed profound central nervous system depression from the $4.5 \mathrm{~g} /$ night dosage [15]. In one case there was associated vomiting and fecal incontinence; the other case was treated with endotracheal intubation but recovered and was discharged the same day. These cases appear consistent with a dose characterized by Erowid in the range of "heavy recreational" to "overdose." For a 70-kg adult, a 4.5-g sodium oxybate dose corresponds to the ingestion of $45.95 \mathrm{mg} / \mathrm{kg}$ of $1,4-\mathrm{BD}$.

From these calculations, it is expected that to achieve a 1,4-BD "overdose" from the ingestion of Aqua Dots beads, the total dose would need to be in the 46-124 mg/ kg range. Obviously, larger doses would still cause overt toxicity, while doses slightly below the lower end of this range might still cause clinical effects that would prompt medical attention. Since Aqua Dots contain $10.8 \mathrm{mg}$ 1,4-BD per bead, this corresponds to the ingestion of $4.26-11.48$ beads $/ \mathrm{kg}$. To give a concrete example, a 2 -year-old toddler weighing $12 \mathrm{~kg}$ would have to ingest roughly 50-140 Aqua Dots beads to overdose on 1,4-BD. Because the beads are very small (and attractively colored) such an ingestion could be easily accomplished; 140 Aqua Dots beads could be packed into a volume less than $15 \mathrm{~mL}$.

Most of the children reported with adverse effects from ingesting Aqua Dots beads were very young. Seven out of 9 patients with their ages reported were toddlers, aged 14, 17, 18, 19, and 20 months, and a two-year-old $[2,4-9,16]$. The 2 other children were 7 and 10 years old $[9,14]$. Aqua Dots was marketed for children aged 4 years and up and was labeled as a potential choking haz- 
ard. Such warnings clearly cannot prevent younger children from playing with and ingesting parts of their older siblings' toys. The 10-year-old child had a history of Asperger's syndrome and attention deficit hyperactivity disorder, which may have placed her at greater risk for ingestion despite age-appropriateness of the toy [9]. This child was later able to conduct a coherent television interview, so her intellectual limitations appeared minimal. After she recovered, the 7-year-old child stated that she thought the beads ". . . were sweets . . . and they had tasted of marzipan" [14].

In only a few cases were the number of ingested beads reported, and some of these were only rough estimates. A 20-month-old American boy ingested "several dozen beads," which resulted in a 6-hour-long coma [4,7]. A 2-year-old Australian boy "swallowed large amounts ... and became seriously ill" [5]. This same patient was included in a 2-patient case series in the medical literature, in which he was reported to have "passed a substantial number of coloured beads in his stool" after recovering from a 7-hour-long coma, but did not estimate the number of beads ingested [9]. The 10-year-old girl had been unarousable for about an hour, then "vomited up to 100 Bindeez beads," followed by a seizure that prompted medical evaluation. She was alert and communicative 5 hours after the ingestion [9]. The 7-year-old patient, who suffered respiratory arrest and was endotracheally intubated for less than 24 hours, later stated that she had eaten about 80 beads [14]. This last case, which occurred in London, appears to provide the best estimate of number of beads ingested. Her estimated ingested 1,4-BD dose was $864 \mathrm{mg}$, and assuming her weight was average ( 23 kg) this corresponds to about $37 \mathrm{mg} / \mathrm{kg}$. This is somewhat less than the predicted necessary dose of $46-124 \mathrm{mg} / \mathrm{kg}$. She may have been particularly sensitive, or her toy set contained more 1,4-BD per bead, or perhaps she underestimated the number of beads she had eaten. Another study group from London has analyzed Bindeez beads procured locally with GC-MS and presented their data in abstract form [17]. They found that 1,4-BD was present in concentrations of "greater than $10 \mathrm{mg}$ " per bead, without any GHB or $1,5-\mathrm{PD}$, which is consistent with our results.

\section{CONCLUSIONS}

Aqua Dots beads contained $13.7 \pm 2.4 \%$ of extractable $1,4-\mathrm{BD}$ by weight. No 1,5-PD was detected, corroborating reports that this benign chemical had been completely replaced with a potentially toxic substitute that is metabolized into GHB after ingestion. Reports of ataxia, vomiting, seizures, and self-limited coma in children are consistent with the ingestion of several dozen Aqua Dots beads.

The authors have no potential financial conflicts of interest to report.

\section{REFERENCES}

1. Mirpuri D [webpage on the Internet]. Hot Toys of 2007Toy Wishes Hot Dozen [updated 2007 Oct 11; cited 2008 Nov
22]. Available from: http://toys.about.com/b/2007/10/11/ hot-toys-of-2007-toy-wishes-hot-dozen.htm

2. Lane $S$ [webpage on the Internet]. Bindeez toy banned among GHB drug claim [updated 2007 Nov 6; cited 2008 Nov 22]. Available from: http://www.abc.net.au/worldtoday/content/ 2007/s2082999.htm

3. Pilkington E, Pallister D [webpage on the Internet]. Global scare after ecstasy-like chemical found in toy [updated 2007 Nov 8; cited 2008 Nov 22]. Available from:

http://www.guardian.co.uk/world/2007/nov/08/china.australia

4. U.S. Consumer Product Safety Commission [webpage on the Internet]. Spin Master Recalls Aqua Dots-Children Became Unconscious After Swallowing Beads [updated 2007 Nov 7; cited 2008 Nov 22]. Available from:

http://www.cpsc.gov/cpscpub/prerel/prhtml08/08074.html

5. Australian Broadcasting Corporation News [webpage on the Internet]. Bindeez banned over GHB fears [updated 2007 Nov 6; cited 2008 Dec 2]. Available from: http://www.abc.net.au/news/stories/2007/11/06/2082480.htm

6. McCrady M, George M, Leamanczyk L [webpage on the Internet]. Exclusive: Local Child Hospitalized After Ingesting Aqua Dots [updated 2007 Nov 8; cited 2008 Dec 2]. Available from: http://www.todaystmj4.com/news/local/11107146.html

7. Harris P [webpage on the Internet]. Aqua Dots latest Chinese-made toy pulled off shelves [updated 2007 Nov 9; cited 2008 Dec 2]. Available from: http://findarticles.com/p/ articles/mi_qn4176/is_/ai_n21104923

8. Bradsher K [webpage on the Internet]. Sleuthing for a danger in toy beads [updated 2007 Nov 8; cited 2008 Dec 2]. Available from: http://www.nytimes.com/2007/11/08/business/ worldbusiness/08recall.html

9. Gunja N, Doyle E, Carpenter K, et al. $\gamma$-Hydroxybutyrate poisoning from toy beads. Med J Aust 2008:188:54-55.

10. Zvosec DL, Smith SW, McCutcheon R, et al. Adverse events, including death, associated with the use of 1,4-butanediol. $N$ Engl J Med 2001;344:87-94.

11. The Vaults of Erowid [webpage on the Internet]. GHB Basics [updated 2007 May 28; cited 2008 Dec 1]. Available from: http://www.erowid.org/chemicals/ghb/ghb_basics.shtml

12. The Vaults of Erowid [webpage on the Internet]. GHB Effects [updated 2007 May 28; cited 2008 Dec 1]. Available from: http://www.erowid.org/chemicals/ghb/ghb_effects.shtml

13. The Vaults of Erowid [webpage on the Internet]. GHB Dosage [updated 2007 May 28; cited 2008 Dec 1]. Available from: http://www.erowid.org/chemicals/ghb/ghb_dose.shtml

14. Runnacles JLM, Stroobant J. Poisoning from toy beads. BMJ 2008;336:110.

15. Jazz Pharmaceuticals. Package insert for Xyrem (sodium oxybate) oral solution.

16. Squidoo [webpage on the Internet]. Aqua Dots [cited 2008 Dec 3]. Available from: http://www.squidoo.com/aquadots

17. Davies S, Button J, Dargan PI, et al. Analysis of biological samples and beads from a case of Bindeez beads (Aqua Dots) ingestion [abstract]. Clin Toxicol 2008;46:596. 